\title{
Einige Spezifika der Psychotherapie mit international Schutzsuchenden
}

\author{
Ruth Kronsteiner (D)
}

Eingegangen: 16. Januar 2017 / Angenommen: 28. April 2017 / Online publiziert: 23. Mai 2017

(c) Der/die Autor(en) 2017. Dieser Artikel ist eine Open-Access-Publikation.

Zusammenfassung Flucht ist ein traumatisierender Prozess und das Asylverfahren zumindest eine Sequenz davon. Wenn Menschen, die um internationalen Schutz ansuchen, in Psychotherapie kommen, befinden sie sich in einer akut traumatisierenden Situation und nicht in Sicherheit. Dafür bedarf es einiger Modifikationen des psychotherapeutischen Rahmens und der Arbeitsweise. Die Sicherheit des Rahmens bekommt somit eine besondere Bedeutung und Wichtigkeit.

Schlüsselwörter Asylverfahren · Sequentielle Traumatisierung · Der „sichere Rahmen“ der Psychotherapie • Dolmetschunterstützte Psychotherapie · Psychotherapeutische Stellungnahmen im Asylverfahren

\section{Some specifics of psychotherapy with international asylum seekers}

Summary Escape is a traumatizing process and the asylum procedure is at least a sequence of it. When people who apply for international protection come to psychotherapy, they are in an acute traumatizing situation and not safe. This requires some modifications of the psychotherapeutic framework and the method. The safety of the frame gets therefore a further meaning and importance.

Keywords Asylum procedures - Sequential traumatization - The "safe framework" of psychotherapy · In-

Diese Arbeit ist Teil des Leitthemas „Traumatherapie in der Praxis“.

R. Kronsteiner $(\bowtie)$

Psychotherapeutische Praxis, Windmühlgasse 21, 1060 Wien, Österreich

ruth.kronsteiner@chello.at terpreting-assisted psychotherapy $\cdot$ Psychotherapeutic expert opinions in the asylum procedure

\section{Flucht als traumatisierender Prozess}

„Flüchtlinge“ entsprechend der Genfer Flüchtlingskonvention bezeichnen sich selbst als „Exilierte“, um die politische Ursache für ihre Migration und den damit verbundenen „Entwertungsprozess“ (FelberVillagra 1995) in den Vordergrund zu stellen. Der Begriff „Schutzsuchende“ betont die internationale Verpflichtung der Zielländer Schutz zu gewähren und wird hier in diesem Sinne verwendet. „Flüchtling“ ist eine Person, die ,aus der begründeten Furcht vor Verfolgung wegen ihrer Rasse, Religion, Nationalität, Zugehörigkeit zu einer bestimmten sozialen Gruppe oder wegen ihrer politischen Überzeugung sich außerhalb des Landes befindet, dessen Staatszugehörigkeit sie besitzt, und den Schutz dieses Landes nicht in Anspruch nehmen kann oder wegen dieser Befürchtungen nicht in Anspruch nehmen will“ (Genfer Flüchtlingskonvention 1951; www.asyl.net 2017). Viele Schutzsuchende würden demnach internationalen Schutz zuerkannt bekommen müssen, dem ist real nicht so. Die Rechtslage zum Asylwesen ist komplex und trotz internationaler und EU- rechtlicher Judikatur von nationalstaatlichen und politischen Eigenheiten geprägt. Die Menschen, die in Österreich um internationalen Schutz ansuchen, kommen derzeit (2016) hauptsächlich aus Afghanistan und Syrien, wobei die Antragsstellungen seit Mai 2016 im Vergleich zum Vorjahr stark rückläufig sind. Die meisten unbegleiteten minderjährigen Flüchtlinge stammen aus Afghanistan. Die Anerkennungsquote als Konventionsflüchtling betrug 2016 bei Personen aus Afghanistan $24 \%$ und bei Menschen aus Syrien $89 \%$ (BM.I Republik Österreich 2017; www.bmi.gv.at). 
Die Flucht selbst ist ein traumatisierender Prozess. Das Bedeutende an diesem Verständnis von Trauma ist, dass nicht alleine das Ereignis katastrophalen Ausmaßes die Ursache für die Traumatisierung darstellt, sondern der damit einhergehende soziale Prozess. Diese Erkenntnis hat Auswirkungen auf die psychotherapeutische Behandlung. Wenn PsychotherapeutInnen mit Schutzsuchenden arbeiten, bedeutet dies, dass sich diese in einer traumatisierenden Situation befinden. Das Trauma geht weiter, was behandlungstechnische Relevanz hat. In der Psychotherapie werden Menschen durch ein Asylverfahren begleitet, das psychisch hochgradig belastend sein kann, je nachdem, ob es im Verfahren zu Re- Traumatisierungen oder Wiederholungen von traumatischen Erfahrungen kommt. Es besteht die rechtliche Möglichkeit Verfahren wohlwollend, schonend, entsprechend der juristischen Standards zur Glaubwürdigkeitsprüfung bei Traumatisierung durchzuführen. Das Erlittene kann respektiert werden, ohne dass Asyl gewährt werden muss. Dies erfolgt häufig nicht. Schutzsuchende werden entwertend und destruktiv in den Verfahren behandelt. Die Haltung der BehördenvertreterInnen zum Menschen an sich, also deren Menschenbild, lässt oft zu wünschen übrig.

In der Psychotherapie mit geflüchteten Menschen können folgende Gruppen unterschieden werden: 1. Jene, die im Zulassungs- oder Asylverfahren sind. Im Zulassungsverfahren wird die Zuständigkeit für das Asylverfahren jenes EU- Mitgliedstaates, in dem der Antrag auf Asyl eingebracht wurde, geprüft. 2. Jene, die endgültig keinen Schutz bekommen und in Schubhaft genommen werden, wenn sie nicht freiwillig zurückkehren. 3. Jene, die Schutz entsprechend der Genfer Flüchtlingskonvention bekommen. 4. Jene, die subsidiären Schutz aber kein Asyl erhalten, weil sie nicht in ihre Herkunftsländer zum Beispiel aufgrund fehlender Rücknahmeabkommen abgeschoben werden können. 5. Jene, die bereits die Staatsbürgerschaft des Aufnahmelandes haben. 6. Jene, die als Kind geflüchtet sind. Jene Menschen, die von der transgenerationalen Weitergabe des Traumas betroffen sind, könnten als siebente Gruppe eingestuft werden. Dazu gehören auch die Nachkommen der autochthonen Bevölkerung, die vom Nationalsozialismus oder vom Zweiten Weltkrieg betroffen waren.

Hans Keilson (2005 [1979]) erkannte und verdeutlichte in seinem Buch „Sequentielle Traumatisierung bei Kindern“, in dem er die Traumatisierung jüdischer Kriegswaisen als Prozess verstand, die Bedeutung der „Soziogenese“ neben der „Psychogenese“ für extreme Traumatisierung. Er spricht von einer „Gliederung der extremen Belastungssituation in drei traumatische Sequenzen“ (Keilson 2005 [1979], S. 56).

Die erste Sequenz ist von der beginnenden Verfolgung zum Beispiel einer ethnisierten Gruppe, der negative Eigenschaften zugeschrieben werden, gekennzeichnet. Die „ängstliche Erwartungsspannung“ (Keilson 2005 [1979], S. 57) der Betroffenen steigt. Der
Rechtsschutz für Betroffene bröckelt ab. Deren wirtschaftliche Existenz wird vernichtet. Menschen verschwinden. Würde und Integrität werden zerstört. Die Ghettoisierung setzt ein. Die Verfolgten werden von der nicht verfolgten Gemeinschaft getrennt und isoliert. Die vertraute Umgebung wird aufgelöst.

Die zweite Sequenz beschreibt die direkte Gewalt und Vernichtung. Die „traumatogenen Momente“ (ebda.) treten deutlich zutage. „Sie enthalten neben der direkten Lebensbedrohung, der Rechtlosigkeit [...], dem Ausgeliefertsein an eine feindliche Umgebung, die im stressorischen Sinne zu verstehenden Dauerbelastungen wie [...] Hunger, Krankheit; ferner [...] Zermürbung, Infragestellung und Vernichtung mitmenschlicher Verhaltensweisen - gemessen an überlieferten und kulturell gesichert erscheinenden Normen - durch die Konfrontation mit der brutalen Macht, dem Grauen und dem Tod“(ebda.).

Die dritte traumatische Sequenz ist für Keilson von besonderer Bedeutung, da sie die Rückkehr in die Rechtsstaatlichkeit bedeutet. Es ist die Rückkehr aus der Rechtlosigkeit in Rechtssicherheit und somit in geordnete und sichere Zustände. Es ist die Phase nach der Verfolgung, nach dem Krieg, nach dem Gefängnis, nach der Folter. Es ist die Phase in der die rechtliche, soziale und medizinische Rehabilitation stattfinden sollte. Kommt es nicht zu einer zweifelsfreien Anerkennung der Opferschaft und zu einer vollständigen Rehabilitation setzt sich die extreme Belastung fort und neue Schädigungen der Betroffenen folgen. Dieser Phase ist das Asylverfahren zuzuordnen.

Flucht bedeutet ebenfalls einen traumatisierenden Prozess zu durchlaufen. Die Flucht selbst stellt eine extreme Belastung dar, die in Anlehnung an Keilson in sechs Sequenzen (Becker 2006) unterteilt wird:

1. In der Zeit vom Beginn der Verfolgung bis zur eigentlichen Flucht setzt die systematische Verfolgung von Menschen ein, die als Gruppen (JüdInnen, TschetschenInnen, YesidInnen, Frauen, Widerständige, ...) konstruiert werden. Menschen werden schikaniert, vertrieben, inhaftiert, gefoltert und getötet. Gefolterte, die in ihr soziales Umfeld zurückkehren, wirken wie ein Mahnmal für die Anderen. Meist wird über das Erlittene geschwiegen, trotzdem wissen alle Bescheid. Angst und Schrecken verbreiten sich in der Gruppe. In dieser Situation gilt es, die Gefahr zu erkennen, die Flucht vorzubereiten und durchzuführen.

2. Die Flucht ist gefährlich und findet im Illegalen statt. Es gibt derzeit keine EU-rechtliche Möglichkeit „legal“ zu fliehen (bis 2001 gab es das „Botschaftsasyl“, d. h. Verfolgte flüchteten in die nächstgelegene Botschaft, in der ihnen Asyl gewährt und die legale Einreise in das Aufnahmeland ermöglicht wurde. So geschehen zum Beispiel in Chile 1973). Flucht bedeutet „FluchthelferInnen“ ausgeliefert zu sein und sich in lebensbedrohlichen Situationen zu befinden. Wie zum Beispiel in engen Räumen 
eingeschlossen $\mathrm{zu}$ sein, wartend wieder rausgelassen zu werden, wenig Luft zum Atmen, kein Essen, nichts zu Trinken und keine Möglichkeit zur Verrichtung der Notdurft zu haben oder auf einem überfüllten Boot ohne Bootsführer zu sitzen. Lange Fußmärsche, sich verstecken, Fahrten unter Lastwägen an der Bodenplatte hängend gehören dazu. Aber auch misshandelt und vergewaltigt zu werden, zuzusehen wie andere misshandelt, vergewaltigt und getötet werden, zählen zu den schrecklichen Erlebnissen auf der Flucht. Frauen und Kinder sind besonders gefährdet, sexualisierte Gewalt zu erleiden und in sklavenähnliche Verhältnisse zu geraten. Sich für die Flucht zu entscheiden, bedeutet auch, die Kraft aufzubringen trotz der Gefahren das Ziel zu verfolgen, sich selbst und Andere in Sicherheit zu bringen.

3. Die Anfangszeit am neuen Ort ist vom Asylverfahren und von der einsetzenden „Migrationskrise“ (Kronsteiner 2003) bestimmt. Die Krise ist von der Trauer um das Verlorene, der Verunsicherung der Identität, von der Regression in die Zeit der Vorsprachlichkeit aufgrund des Sprachwechsels sowie vom Zulassen der Gefühle, die mit dem Erlebten verbunden sind, geprägt. Eine Studie (Ammer et al. 2013, S. 172) zeigt, dass selbst Zulassungsverfahren Monate sogar Jahre dauern können, wenn in erster Instanz kein Asyl gewährt wird. Die Erwartungen an das Asylverfahren können über die Gewährung von dauerhaftem Schutz hinausgehen. Oft glauben Schutzsuchende, dass es im Verfahren um Gerechtigkeit geht. Dies würde bedeuten, dass die Behörde dem schutzsuchenden Menschen glaubt, ihn als Opfer von Gewalt und Verfolgung anerkennt sowie ihm Sicherheit gewährt. „Somit hat die Glaubwürdigkeitsfrage im Asylverfahren einen symbolischen Charakter, der mit Anerkennung von getanem Unrecht, Bestrafung der Täter und Wiedergutmachung an den Opfern verbunden ist" (Ammer et al. 2013, S. 48). Die Prüfung der Glaubwürdigkeit hat im Asylverfahren einen zentralen Stellenwert.

4. In der Sequenz der Chronifizierung der Vorläufigkeit zeichnet sich ab, dass eine baldige Rückkehr nicht möglich sein wird. Das Asylverfahren erstreckt sich bereits über Monate oder auch über Jahre. Diese Zeit ist von den Möglichkeiten im Aufnahmeland bestimmt. Die Menschen passen sich an, ihre Identität verändert sich, ob sie wollen oder nicht. Integration findet in jedem Fall statt. Sie kann vom Aufnahmeland unterstützt oder behindert werden. Diskriminierungen, mangelnde Unterstützung beim Spracherwerb und bei der Berufswahl erschweren die Integration.

5. Die Klärung der Rückkehrabsichten bestimmt diese Sequenz. Die Entscheidung zum Verbleib im Aufnahmeland oder zur Rückkehr wird getroffen. Die Rückkehr selbst und die erste Zeit am Herkunftsort gehören ebenfalls zu dieser Sequenz. Die Rückkehr beinhaltet eine neuerliche „Migrationskrise“ zu er- leben. Vieles hat sich am Herkunftsort verändert. Man hat sich selbst durch die Exilierung verändert. Oft bleiben erwachsen gewordene Kinder im Aufnahmeland zurück. Viele entscheiden sich für den Verbleib am Aufnahmeort oder auch für eine neuerliche Migration. Eine erzwungene Rückkehr ist mit Gewalt verbunden und bedeutet neue Traumata $\mathrm{zu}$ erleiden. Im Herkunftsland ist neuerliche Gewalt und unter Umständen der Tod zu erwarten.

6. In dieser Sequenz setzt die eigentliche Rehabilitation ein, nachdem Asyl gewährt und eine relative Sicherheit gewährleistet worden ist. Sowohl in der Herkunftsgesellschaft als auch in der Aufnahmegesellschaft sind der gesellschaftliche Umgang mit dem erlittenen Unrecht, mit der Entwertung durch die Exilierung, der Habhaftmachung der Täter, der Anerkennung der Opferschaft, der öffentlichen Aufarbeitung und Wiedergutmachung bedeutend für den Heilungsprozess von Traumafolgeerkrankungen.

In Österreich gibt es mittlerweile einige Einrichtungen, die adäquate Psychotherapie aber auch Befundungen und ärztliche Versorgung kostenlos für Schutzsuchende anbieten sowie KollegInnen beraten und supervidieren. In Wien beispielsweise sind dies Hemayat - Betreuungszentrum für Folter- und Kriegsüberlebende und das Projekt Sintem der Caritas, in Innsbruck Ankyra - Zentrum für interkulturelle Psychotherapie in Tirol, eine Einrichtung des Flüchtlingsdienstes der Diakonie oder in Graz das interkulturelle Beratungs- und Therapiezentrum Zebra. Einige Organisationen in Österreich haben sich zum „Netzwerk für interkulturelle Psychotherapie nach Extremtraumatisierung“ (NIPE) zusammengeschlossen. Charakteristisch für die Psychotherapie mit Schutzsuchenden ist das Ineinandergreifen von Recht und Psychotherapie.

Nach Judith Herman (2006 [2003]) sind für den Genesungsprozess extrem traumatisierter Menschen eine heilende Beziehung, Sicherheit, das Erinnern und Trauern sowie die Neugestaltung der Zukunft erforderlich. Der Fokus in der ersten Phase der Psychotherapie liegt auf dem Herstellen von Sicherheit. „The alliance between therapist and patient is formed initially around the common goal of ensuring a reasonable degree of safety, and no other work should be attempted until this goal has been achieved. The focus of safety begins with control of the body and moves outward to issues of control of the environment. [...] Environmental issues include the establishment of a safe living situation ..." (Lebowitz et al. 1993, S. 379). Schutzsuchende in laufenden Verfahren sind nicht in Sicherheit. Sie sind zwar der akuten Verfolgung entkommen, müssen aber damit rechnen dorthin zurückgeschickt zu werden. Dies führt zu großer Angst. In der Psychotherapie können eine heilsame Beziehung und ein entsprechendes Arbeitsbündnis angeboten werden. Die medizinische 
Versorgung des Körpers und kompetente rechtliche Betreuung stehen am Beginn der Psychotherapie. Die PatientIn braucht zur Genesung ,stabile Lebensbedingungen, finanzielle Sicherheit, Mobilität sowie einen Plan zum Selbstschutz" (Herman 2006 [2003], S. 223), der alle Aspekte ihres täglichen Lebens miteinbezieht. Dies sind zentrale Themen der Therapie zu Beginn aber auch während des Verfahrens. Aufgrund des unsicheren Aufenthalts und der einzelnen Schritte im Verfahren wie Einvernahmen und Begutachtungen durch Sachverständige treten immer wieder schwere Krisen auf und die Depression scheint unaufhaltsam. Für Schutzsuchende ergibt sich aufgrund von Traumafolgeerkrankungen die Notwendigkeit, psychotherapeutische ExpertInnenmeinungen (Befundberichte, Psychotherapeutische Stellungnahmen, Gutachten) in ihr Verfahren einzubringen, damit sie nach entsprechenden Standards einvernommen werden.

\section{Psychotherapeutische ExpertInnenmeinungen als juristische Beweismittel}

Befundberichte sollten die Anamnesemethoden, die Anamnese, die darin berücksichtigten Befunde, die daraus abgeleiteten Diagnosen und den Therapieplan beinhalten. Aufgrund rechtlicher Implikationen ist davon auszugehen, dass Schutzsuchende im Zulassungs-, Anerkennungs- und Beschwerdeverfahren Befundberichte, psychotherapeutische Stellungnahmen (Darlegung der fachlichen Meinung $\mathrm{zu}$ einem Sachverhalt, die nachvollziehbar, überzeugend und plausibel begründet wird) oder bei Befähigung der PsychotherapeutIn Gutachten benötigen, die die Traumata, Traumafolgeerkrankungen sowie die Einvernahmefähigkeit darlegen und einschätzen. Es gibt Möglichkeiten durch Umschreibungen in den besagten Schriftstücken die Verschwiegenheitspflicht einzuhalten. Erfahrungsgemäß ist die Einschätzung der Authentizität der schutzsuchenden Person, für die Glaubwürdigkeitsprüfung im Verfahren hilfreich, wenn sich die Person schon länger in Psychotherapie befindet. PsychotherapeutInnen arbeiten viele Stunden mit ihren PatientInnen, diese Möglichkeit haben die BehördenvertreterInnen nicht. Wenn durch eine Zurück- oder Abschiebung unzumutbare Verschlechterungen für den Gesundheitszustand $\mathrm{zu}$ erwarten sind und diese somit einen Verstoß gegen die Menschenrechtskonvention darstellt, muss das Verfahren zugelassen werden beziehungsweise ist eine Abschiebung unzulässig. In diesem Fall sollte darauf in einem Befundbericht eingegangen werden. Auch der Eingriff in eine laufende psychotherapeutische Behandlung, der durch eine Beendigung des Aufenthalts gegeben ist, kann zu einer erheblichen Verschlechterung des Gesundheitszustandes führen. Befunde oder Ähnliches sind Beweismittel im Verfahren und können positive Wirkung auf den Verlauf desselben haben. Meist gibt die Behörde aufgrund der von Schutzsuchenden eingebrachten Befundberichte Sachverständigengut- achten in Auftrag. In der bereits erwähnten Studie (Ammer et al. 2013), die Asylverfahren von Folterüberlebenden in Österreich juristisch und psychotherapeutisch untersucht, zeigt sich, dass die subjektive Haltung der Behörde und der behördlich beauftragten GutachterInnen zu den Schutzsuchenden den Verlauf und den Ausgang des Verfahrens bestimmen.

\section{Die Sicherheit des psychotherapeutischen Rahmens}

Der psychotherapeutische Rahmen, der die Frequenz von zum Beispiel einer Einheit pro Woche an einem bestimmten Tag zu einer bestimmten Zeit für die Dauer des Bedarfs der Behandlung regelt, gibt in der unsicheren Lebenssituation des Asylverfahrens Sicherheit, die verinnerlicht wird. Diese Stabilität, die sich auch in der Zuverlässigkeit der TherapeutIn und in klaren Zeiten der Erreichbarkeit aber auch der Nichterreichbarkeit ausdrückt, sich auch zum Beispiel in der korrekten Aussprache des Namens der PartientIn zeigt, ist die erste Voraussetzung für Vertrauen und Sicherheit im therapeutischen Prozess. Gerade in der Psychotherapie mit Schutzsuchenden wird am Rahmen aus unterschiedlichen Gründen gerüttelt, das sich auf die TherapeutIn überträgt. Es ist wichtig, dies zu erkennen, bei der Stabilität des Rahmens zu bleiben und es immer wieder zu besprechen, wenn der Rahmen nicht eingehalten wird. Diese Stabilität ist ein zentraler Faktor in der Psychotherapie mit extrem traumatisierten Menschen besonders in der Zeit der Unsicherheit. Es ist von zentraler Bedeutung für das Gelingen der Therapie, dass sich die TherapeutIn nicht destabilisieren lässt. Dazu gehört auch, dass die Finanzierung der Therapie gesichert ist. Schutzsuchende können meist eine Psychotherapie nicht selbst bezahlen, auch nicht einen Sozialtarif. Dies bedeutet, dass ein Therapieplatz mit voller Kostenübernahme durch Hilfsorganisationen und Kassen erforderlich ist. Schutzsuchende dürfen in der Regel nicht arbeiten und sind über die Grundversorgung sozialversichert. Zur Sicherheit gehört auch, dass die sprachliche Verständigung gewährleistet ist. Oft ist Psychotherapie nur unter Beiziehung von DolmetscherInnen möglich, die ebenfalls bezahlt werden müssen. Die Krankenkassen übernehmen in der Regel diese Kosten nicht. Mittlerweile bieten PsychotherapeutInnen auch in der jeweiligen Muttersprache Psychotherapie an.

\section{Dolmetschunterstützte Psychotherapie}

Die dolmetschunterstützte Psychotherapie erfordert nicht nur einen finanziellen, organisatorischen und zeitlichen Mehraufwand sondern auch die Berücksichtigung der Triade im therapeutischen Setting und Prozess, was schwierig aber auch befruchtend sein kann. Die übersetzende Person braucht nicht nur die Qualifikation zu dolmetschen, sondern auch eine adäquate Schulung für das Übersetzen im psy- 
chotherapeutischen Kontext. Diese beinhaltet eine entsprechende Selbstfürsorge, um Erzählungen über Furchtbares aushalten zu können, sich nicht mit der PatientIn oder mit der TherapeutIn zu identifizieren, sondern sich darauf zu konzentrieren, die "Zunge“ der beiden $\mathrm{zu}$ sein. Die beste Übersetzung ist die, bei der die Anwesenden mit der Zeit nicht mehr wahrnehmen, dass eine Übersetzung stattfindet. Die TherapeutIn braucht nach der Therapiesitzung Zeit, um die Sitzung gemeinsam mit der DolmetscherIn zu reflektieren, mögliche Irritationen in der Sitzung oder auch psychische Überforderungen der übersetzenden Person zu besprechen, sie zu stützen. Die DolmetscherIn ist nicht nur Sprach- sondern auch KulturvermittlerIn und somit eine wichtige Informationsquelle über die „Normalität“ im jeweiligen kulturellen Kontext.

Die Schulung der übersetzenden Person sollte auch beinhalten, dass Äußerungen der PatientInnen im genauen Wortlaut und nicht scheinbar sinnhaft übersetzt werden. „Ob ein Klient in vollständigen Sätzen oder abgehackt spricht, ob häufig Füllwörter zum Einsatz kommen und ob es Besonderheiten oder Befremdliches im Sprachgebrauch gibt. All das kann für den Psychotherapeuten ebenso aufschlussreich sein, wie die inhaltliche Komponente des Gesagten“ schreibt Mascha Dabic, die in Therapien dolmetscht und dies auch lehrt (Dabic 2010, S. 151). Weiter führt sie aus: „Auch das, was nicht gesagt wird, also verschwiegen oder ausweichend beantwortet wird, kann aufschlussreich sein und Material für die Therapeutinnen liefern. In der Psychotherapie müssen Dolmetscherinnen mitunter längere Gesprächspausen „aushalten“ können ...“ (ebda.). Für PsychotherapeutInnen ist dies eine Selbstverständlichkeit, nicht so für DolmetscherInnen. Zusätzlich ist auch für die übersetzende Person die absolute Verschwiegenheit geboten und der Kontakt mit PatientInnen außerhalb der Psychotherapie untersagt. „Im Rahmen der dolmetschunterstützten Psychotherapie hängt der Therapieerfolg daher wesentlich von der Bereitschaft der Dolmetscherinnen ab, sich auf diese heikle Gesprächssituation einzulassen und die Klientinnen im Heilungsprozess zu begleiten“ (Dabic 2010, S. 160). Dolmetschunterstützte Psychotherapie über einen längeren Zeitraum kann auch dazu führen, dass PatientInnen die deutsche Sprache schneller und besser erlernen, was ihnen einen Zugang $\mathrm{zu}$ einer neuen Welt eröffnet. Denn der „... Zugang zu Emotionalität, Phantasien und Symbolen, Identifikationen und Internalisierungen muss sprachlich neu gebahnt und der Kommunikation erschlossen werden - ein aufwändiger, belastender Prozess“ (Kohte-Meyer 2009, S. 150).

\section{Besonderheiten im Erstgespräch}

Bereits bei der Begrüßung können sich kulturelle Unterschiede zeigen, die besprochen werden sollten. $\mathrm{Zu}$ - schreibungen an die PsychotherapeutIn sind von Anfang an virulent und von Erfahrungen im Herkunftsland geprägt. Es wird schnell spürbar, wenn die Psychotherapie wie ein Besuch bei einer „Behörde“ gesehen wird.

Häufig gehören Schutzsuchende zu einer verfolgten Minderheit im Herkunftsland. Die Übersetzung in der Psychotherapie erfolgt entweder in der Muttersprache oder in der Zweitsprache (Amtssprache, die häufig als „Sprache der Macht“ im Herkunftsland erlebt wird) der PatientInnen. Wenn die Übersetzung zum Beispiel auf Russisch und nicht auf Tschetschenisch oder auf Arabisch oder Türkisch und nicht auf Kurdisch erfolgt, ist es wichtig dies im Erstgespräch $\mathrm{zu}$ thematisieren. Das Erzählen in der Zweitsprache ist oft emotional distanzierter als in der Muttersprache, was dazu führt, dass die Gefühle weniger wahrnehmbar, spürbar sind und somit auch zum Beispiel weniger Gefahr durch emotionale Überflutung droht. Übertragungen auf Dolmetschende sind zu beachten und für den therapeutischen Prozess zu nützen. Vorbehalte gegen die dolmetschende oder die therapierende Person sind wichtige Quellen des Verständnisses des Konflikts, der mit dem Trauma verbunden ist. Übertragungen auf diese und Abwehrmechanismen werden erkennbar. Das Erklären von Psychotherapie, der Arbeitsweise, des Settings, der Vertraulichkeit ist wie in jedem Erstgespräch erforderlich, ebenso wie das Abklären des Anliegens und psychotherapeutischer Vorerfahrungen. Hilfreich sind psychotherapeutische Erfahrungen im jeweiligen kulturellen Kontext zu erfragen, die es meist gibt, aber anders benannt werden. Diese offen $\mathrm{zu}$ besprechen ebenso wie die Fluchtgeschichte zu erfassen, nach dem Herkunftsort und den dort Gebliebenen zu fragen, ist für das Herstellen eines Arbeitsbündnisses unerlässlich. Wenn jemand inhaftiert war, kann man davon ausgehen, dass die Person auch gefoltert wurde. Es ist keine Seltenheit, dass Erstgesprächssituationen Erinnerungen an Verhörsituationen und an Situationen psychischer Folter auslösen. Als PsychotherapeutIn die eigene Gegenübertragung und Abwehr zu erkennen und zu reflektieren ist die eigentliche Basis für das Gelingen der Psychotherapie.

\section{Therapieverlauf}

Im Erstgespräch zeigen sich der Leidensdruck, die Motivation zur Therapie, die Abwehrstruktur wie auch unbewusste Konflikte hinter dem Leiden, aber vor allem auch die aktuelle Situation der Schutzsuchenden. Die Unterbringung, die Versorgung, das Arbeitsverbot und ganz besonders die Unsicherheit des Aufenthalts sowie der Verlauf des Asylverfahrens sollten besprochen werden. Die Angst, kein Asyl zu bekommen, im Aufnahmeland nicht bleiben zu dürfen, abgeschoben zu werden, Re-Traumatisierungen und Kränkungen im Verfahren zu erleben, die Wut darüber sowie die 
Trauer über Verluste und der Schmerz durch das Erlittene bedürfen therapeutischer Unterstützung.

In manchen Fällen ist der innere Druck, für das Erlebte Worte zu finden und endlich jemandem das Furchtbare erzählen zu können, so groß, dass die gegenwärtige Realität in den Hintergrund tritt. „Er hatte das Bedürfnis, endlich alles in Worte zu fassen, was ihm angetan wurde und sich einem mitfühlendem Gegenüber, ohne sich schuldig fühlen zu müssen, anvertrauen zu können“ (Kronsteiner 2010, S. 73). Dieser extrem traumatisierte Mann aus Tschetschenien stand im Beschwerdeverfahren und war mit seiner Frau und zwei kleinen Kindern unter widrigsten Bedingungen geflüchtet. Er hat im Laufe der Therapie internationalen Schutz bekommen, begann zu arbeiten und wollte Prothesenmacher werden, um den Kriegsversehrten helfen zu können, wenn er in seine Heimat zurückkehren kann.

\section{Traumafolgeerkrankungen}

Manchmal besteht das vordergründige Motiv, eine PsychotherapeutIn aufzusuchen darin, einen Befund für das Verfahren zu bekommen. Das ist ein wichtiges Anliegen und offen zu besprechen. Von großer Bedeutung sind die Diagnosen im Befund. Extrem oder kumulativ traumatisierte Menschen können an unterschiedlichen Traumafolgeerkrankungen leiden und nicht unbedingt an einer Posttraumatischen Belastungsstörung (PTBS). Zum Beispiel können depressive, dissoziative und somatoforme Störungen, Angststörungen, Psychosen, Persönlichkeitsveränderungen und somatische Erkrankungen wie Diabetes, Asthma, Hauterkrankungen, Herzrhythmusstörungen, Epilepsie Folgen von Traumatisierung sein. Dies ist für PsychotherapeutInnen nichts Neues, aber für die Asylbehörde oft schon und deshalb im Falle einer Befundung ausführlich zu erklären. Ebenso wichtig ist die Methode der Diagnostik darzulegen und die Anzahl der abgehaltenen Therapiestunden anzuführen.

\section{Einvernahmefähigkeit}

Im Falle bevorstehender Einvernahmen macht es Sinn mit den PatientInnen über deren Einvernahmefähigkeit zu sprechen. Viele wollen so schnell wie möglich ihre Geschichte erzählen, um das Verfahren zu beschleunigen, da das lange Warten zermürbend ist. Die Einschätzung der TherapeutInnen kann gegen eine Einvernahme zum gegebenen Zeitpunkt sprechen, beispielsweise weil eine kohärente affektauthentische Erzählung über die fluchtauslösenden Traumata nicht möglich ist. Gefühle werden abgespalten, die Erzählung wirkt gefühlsarm im Verhältnis zum Inhalt der Erzählung, was als unglaubwürdig bewertet werden kann. Vermeidung führt dazu, dass schreckliche Erlebnisse nicht erzählt werden, um eine Symptomvirulenz wie Erinnerungseinbrüche und Überflutung in der Einvernahmesituation zu vermeiden. In Psy- chotherapien mit Schutzsuchenden geht es häufig darum, die Einvernahme durch die Asylbehörde vorzubereiten. Das kann bedeuten, dass Schutzsuchende das Schweigen erstmalig beenden, für das Unaussprechliche Worte suchen und die Gefühle zulassen, die damit verbunden sind, auch wenn Überflutung droht. Die PsychotherapeutIn hat auch die Aufgabe bei der Suche nach passenden Worten behilflich $\mathrm{zu}$ sein. Hilfreich ist es, die Gefühle wahrzunehmen, die drohende Überflutung zu spüren und zu lernen dies dem Gegenüber in der Einvernahme mitzuteilen. Ebenso verhält es sich mit der Abspaltung der Affekte, wahrzunehmen, dass die zugehörigen Gefühle nicht spürbar sind und dies, gegebenenfalls auch $\mathrm{zu}$ erklären.

\section{Glaubwürdigkeitsprüfung}

In der Glaubwürdigkeitsprüfung spielen korrekte Zeitund Ortsangaben eine bedeutende Rolle. Wenn zum Beispiel vor dem fluchtauslösendem Trauma bereits ein anderes Trauma erfolgt ist, wird nicht selten spontan auf die Frage, in welchem Jahr das traumatische Ereignis passiert sei, das Jahr des ersten Traumas genannt. Die genannte Jahreszahl steht zu den sonstigen Angaben im Widerspruch und die Person wird als unglaubwürdig eingestuft und kein Asyl gewährt. Wenn die Einvernahme trotz einer negativen Einschätzung der TherapeutIn stattfindet, ist es wichtig im Befund oder der Stellungnahme die mögliche Abwehr der PatientIn darzulegen und auf die Gefahren der Re-Traumatisierung hinzuweisen. Oft beauftragt die Behörde ein Gutachten, auf das man als behandelnde PsychotherapeutIn reagieren muss. In der erwähnten Studie (Ammer et al. 2013) zeigte sich, dass es zu regelrechten „Gutachterschlachten“ kommen kann. Behördlich beauftragte GutachterInnen halten sich manchmal weder an fachliche noch an ethische Richtlinien der Gesundheitsberufe, wie sie gesetzlich und von Berufsverbänden festgelegt sind. „Das klinisch-psychologische Gutachten selbst ist ein Ausdruck von Abwehr in Form von Entwertung. Es wirkt wie ein „Gefälligkeitsgutachten“ für die Behörde, die darauf abzuzielen scheint, der Asylsuchenden einen negativen Bescheid geben zu können“ (Ammer et al. 2013, S. 90). Die Glaubwürdigkeitsprüfung ist wohl der bedeutendste Part im bereits zugelassenen Verfahren.

Wenn einem nicht geglaubt und eine negative Entscheidung von der Behörde getroffen wird, ist dies nicht nur sehr bedrohlich, sondern auch schmerzhaft. Es ist keine Seltenheit, dass aus Opfern TäterInnen zum Beispiel aus WiderstandskämpferInnen TerroristInnen - gemacht werden. Opfer sexualisierter Gewalt, besonders Frauen, bringen ihre schrecklichen Erlebnisse häufig nicht sofort oder gar nicht im Verfahren ein. Sie schämen sich, fühlen sich schmutzig und haben Angst, dass Familienangehörige davon erfahren. Wenn die erlebte Gewalt nicht geglaubt wird, ist dies sehr kränkend und löst Ohnmacht aus. Das Hinter- 
fragen des Erlebten durch die Behörden erfolgt häufig in verhöhnender und aggressiver Weise. Dies gilt es, neben vielem anderen, in der Psychotherapie zu verarbeiten, so auch die langen Wartezeiten in den Verfahren.

\section{Lange Wartezeiten nützen}

Asylsuchende warten oft jahrelang, bis sie Asyl zugesprochen bekommen, was unweigerlich zu schweren Depressionen verbunden mit Suizidalität führt und auch stationäre Aufenthalte notwendig machen kann. In den meisten Fällen ist zumindest eine psychiatrische Behandlung parallel zur Psychotherapie erforderlich. Es gilt die Wartezeit zu nützen, trotz Angst und psychischer Erkrankungen Deutsch zu lernen, Ausbildungs- und Berufswünsche zuzulassen sowie die Integration in die Aufnahmegesellschaft voranzutreiben und diese zu dokumentieren, um gegebenenfalls zumindest einen Aufenthalt aus humanitären Gründen erwirken zu können. Möglichkeiten zu überlegen, die im Falle eines Abschiebebescheids vorhanden sind, geben Sicherheit, reduzieren die Angst, eröffnen Handlungsspielräume, helfen gegen die Ohnmacht.

Ohnmacht auszuhalten, Angst nicht abzuwehren, Wut, Verzweiflung, Hoffnungslosigkeit und Trauer anzusprechen und zu halten, Worte für das Unaussprechliche $\mathrm{zu}$ finden und $\mathrm{zu}$ geben, das Furchtbare als solches $\mathrm{zu}$ benennen, sind bedeutende Anforderungen an die TherapeutIn. Quellen der Freude, der Stärkung des Selbstwertes auch des Triumphes sowie der Geborgenheit und Sicherheit in dieser Zeit der äußeren nach wie vor traumatisierenden Situation zu finden, sind ebenfalls charakteristisch für die Psychotherapie mit Schutzsuchenden.

Die Alltagsbewältigung ist in dieser Zeit keine Selbstverständlichkeit. Das Geschrei von Kindern löst Erinnerungen an die Schreie der gequälten Menschen im Lager oder im Gefängnis aus, das Quietschen der Straßenbahn auf den Schienen erinnert an das Quietschen der Metalltüre beim Öffnen, wenn man zur Folter geholt wurde. Intrusionen und dissoziative Zustände sind die Folge, was auch zu gefährlichen Situationen zum Beispiel im Straßenverkehr führen kann. Die Bearbeitung unbewusster Wiederholungen traumatischer Erlebnisse und der damit verbundenen Konflikte gehören zu jeder Traumatherapie.

Schutzsuchenden fehlt die Sprache des Aufnahmelandes, alles muss von irgendjemandem übersetzt werden, Angehörige sind zurückgeblieben und in Gefahr, die Pflichten und Rechte auch das Asylrecht im neuen Land sind unbekannt. Das bedeutet, viele Informationen $\mathrm{zu}$ geben, an weitere HelferInnen $\mathrm{zu}$ vermitteln, sowie unter Umständen einen kompetenten Rechtsbeistand zu organisieren. Dies dient der psychischen Stabilisierung.

Manchmal sind auch Interventionen in der Grundversorgungsunterkunft nötig, da sich auch BetreuerInnen re-traumatisierend verhalten. Die Unterbringung in Lagern birgt große Schwierigkeiten und Gefahren. Neben menschenunwürdigen Zuständen in Flüchtlingslagern (keine Möglichkeit selbst zu kochen, keine Intimsphäre) sind Schutzsuchende auch Gefahren ausgesetzt, die von anderen Geflüchteten ausgehen, wie die Begegnung mit dem eigenen Folterer oder einem verfolgenden politischen Gegner. Vergewaltigte Frauen werden von anderen Geflüchteten bedroht, weil sie als „entehrt“ gesehen werden, um nur einige Beispiele zu nennen. Entlegene Unterkünfte führen zu Intrusionen, da sie an den Ort erinnern, an den man verschleppt, mit dem Tod bedroht worden ist und schwere Gewalt erlitten hat. Abgesehen davon, dass in solchen Unterkünften der Zugang zur Gesundheitsversorgung mit adäquater Übersetzung und zu Bildungseinrichtungen nicht gegeben oder sehr erschwert ist.

Das bewusste Agieren der PsychotherapeutInnen in der Außenwelt der Schutzsuchenden stellt eine therapeutische Herausforderung dar. Die Entscheidung für eine Intervention im Außen ist notwendig, wenn eine Gefahr für die PatientIn besteht, die von ihr selbst nicht abgewendet werden kann. Damit geht auch eine „Infantilisierung“ der PatientInnen einher, die bereits aufgrund der Machtlosigkeit als „Flüchtling“, der Unterbringung in Heimen und der Ohnmacht aufgrund der „Sprachlosigkeit“ in der Aufnahmegesellschaft entsteht. Das Agieren der TherapeutIn hat Auswirkungen auf die therapeutische Beziehung. Der TherapeutIn kann eine Macht zugeschrieben werden, die sie nicht hat. Erfolgreiche Interventionen können zu Idealisierungen, erfolglose zu Entwertungen führen. Jedenfalls können sie Angst machen. Die Transparenz der geplanten Kontakte und deren Ergebnisse sowie die Übersetzung verfasster Schriftstücke helfen, die Angst zu reduzieren, Vertrauen zu stärken, die Sicherheit zu erhöhen. Das Einhalten der Verschwiegenheitspflicht kann dabei eine Herausforderung sein.

Die Community in der Diaspora kann sehr hilfreich aber auch belastend sein, vor allem wenn deren Mitglieder ebenfalls schwer traumatisiert sind. Traumatisierte Menschen tendieren ihr Trauma unbewusst zu wiederholen, es weiterzugeben. Sie können zum Beispiel in der Familie aber auch gegen unbekannte Personen gewalttätig werden, wenn sie sich von diesen entwertet fühlen oder sich in einem schwer dissoziativen Zustand befinden. Dann kann auch die Erinnerung an die Gewalttat dissoziiert und somit nicht erinnerbar sein.

\section{Auswirkungen von Bescheiden auf die Psychotherapie}

Wenn Bescheide zur Gewährung von Asyl in der Zeit der Therapie eintreffen, haben diese natürlich Auswirkungen auf den Verlauf. Bei negativen Bescheiden geht es nicht nur um die ausgelösten Gefühle, sondern um Überlegungen auf der Handlungsebene, um die 
Angst in den Griff zu bekommen. Zuerst sollte der Bescheid detailliert übersetzt und besprochen werden. Bescheide können an die 100 Seiten in juristischem Deutsch umfassen, nur der Beschluss wird in einer für die Schutzsuchenden verständlichen Sprache verfasst. Gespräche mit der Rechtsvertretung können erforderlich sein, um die weiteren juristischen Schritte zu erfahren und eine notwendige Befundung und deren Inhalte zu besprechen. Da eine Beschwerde gegen einen Bescheid nur innerhalb von zwei Wochen möglich ist, muss rasch gehandelt werden. Eine Zurückschiebung in einen EU-Mitgliedsstaat wird oft wie eine Abschiebung in den Herkunftsstaat erlebt, zum einen weil bestimmte Mitgliedsstaaten real kaum Asyl erteilen und die Abschiebung somit nur aufgeschoben ist, zum Anderen fühlen sich viele in jenen Staaten aufgrund politischer Naheverhältnisse zum Herkunftsstaat, aktuell oder historisch, nicht sicher. Zum Beispiel flüchteten viele TschetschenInnen nach Österreich, weil sie sich in den ehemaligen Mitgliedsstaaten des Warschauer Pakts Tschechien, Polen, Ungarn, Slowakei nicht sicher fühlten.

Eine Abschiebung in den Herkunftsstaat aufgrund eines negativen Bescheids kann lebensbedrohlich sein, sonst wäre man nicht geflohen. Schutzsuchende kennen die Situation in ihrem Herkunftsstaat. Es ist wichtig als TherapeutIn zuzulassen, gemeinsam Bilder davon $\mathrm{zu}$ entwickeln, welche Gefahren und welche Handlungsmöglichkeiten bestehen, um sich selbst und andere zu schützen. Um die Realität einschätzen zu können, sind auch Informationen über das Herkunftsland einzuholen.

Menschen, die subsidiären Schutz und kein Asyl gewährt bekommen, wie es bei vielen AfghanInnen der Fall ist, müssen jährlich um Verlängerung ansuchen. Dies bedeutet kurzfristig Sicherheit, aber keine nachhaltige Auflösung der Angst. Allerdings gibt es Erfahrungswerte: Menschen aus bestimmten Regionen bekommen meist problemlos Verlängerungen. Subsidiär Schutzberechtigte dürfen arbeiten und sich in Österreich frei bewegen. Das Ziel ist, sich innerhalb von fünf Jahren so zu „integrieren“, dass eine Abschiebung aufgrund der Europäischen Menschenrechtskonvention nicht mehr möglich ist und eine ordentliche Aufenthaltsgenehmigung erteilt werden muss.

\section{Wenn Schutz gewährt wird}

Wenn nach einem langen Verfahren Asyl gewährt wird, sind die Betroffenen selbst oft zu erschöpft und zermürbt, um sich freuen zu können. Sie sind erleichtert und die Angst ist geringer. Die Medikamente können reduziert werden. Manchmal kommt es zum psychischen Zusammenbruch, weil Gefühle wieder gespürt werden können, die davor abgespalten waren. Anerkannte Konventionsflüchtlinge müssen sich auf ihr neues Leben konzentrieren. Sie beenden die Therapie, nicht weil sie die für sie wichtige Traumaverarbeitung nicht wünschen, sondern weil sie ihre Kraft für den Aufbau ihres neuen Lebens brauchen. Konventionsflüchtlinge können nach sechs Jahren Aufenthalt bei entsprechender „Integration“ die österreichische Staatsbürgerschaft beantragen, die die größte Sicherheit bietet. „Integration“ bedeutet in diesem Fall, die deutsche Sprache bis zu einem bestimmten Niveau nachweislich erlernt, ein eigenes Einkommen und eine Wohnung zu haben, sowie unbescholten $\mathrm{zu}$ sein.

Ältere ehemalige Konventionsflüchtlinge kommen nach Erhalt der Staatsbürgerschaft erstmals oder wieder in Therapie. Der Aufbau des neuen Lebens ist vollbracht, aber das nicht ausreichend bearbeitete Trauma wird erfahrungsgemäß nach einer maximalen „Inkubationszeit“ von 20 Jahren virulent, häufig durch ein äußeres Ereignis. Das Trauma bringt verstärkt Symptome bis hin zur Psychose hervor. Die durch die Staatsbürgerschaft erlangte Sicherheit ermöglicht die Traumaverarbeitung.

Junge Erwachsene, die als Kind flüchten mussten, kommen in Psychotherapie, weil sie Symptome entwickeln, die ihnen rätselhaft erscheinen. Diese werden mit ihren frühen Fluchterfahrungen und den Traumata der Eltern in Verbindung gebracht. Kinder und nachfolgende Generationen erleben das Trauma der Eltern, Großeltern und noch weiter zurückliegender Generationen als „organisierenden Faktor in ihrem Leben“ (Bohleber 2000, S. 815) und als identitätsstiftend.

\section{Schlussfolgerungen}

Die Herausforderungen in der Psychotherapie mit Menschen, die internationalen Schutz suchen, bestehen auch darin, sich mit asylrechtlichen Belangen auseinanderzusetzen. Die Vorbereitung auf Einvernahmen und Begutachtungen dienen sowohl der Verhinderung von Re- Traumatisierungen und Symptomvirulenzen als auch der für die Heilung notwendigen Sicherung des Aufenthalts. Die Lebenssituation von Schutzsuchenden ist per se instabil, da sie sich nach wie vor in einer traumatisierenden Situation befinden. Umso größere Bedeutung kommt der Psychotherapie als „stabiler“ und „stabilisierender“ Faktor zu. Die Notwendigkeit in der Außenwelt der PatientIn zu intervenieren und dies transparent $\mathrm{zu}$ machen, gehört ebenso dazu, wie die Bereitschaft selbst Sprachen zu lernen und mit DolmetscherInnen zu arbeiten. Die Analyse der spezifischen Übertragung und der Auswirkungen der Triade in der dolmetschunterstützten Psychotherapie stellt eine weitere Herausforderung dar. Die Reflexion der PsychotherapeutIn eigener schichtspezifischer und kultureller Stereotypen ist eine Voraussetzung für das Gelingen der gemeinsamen Arbeit. Die Psychotherapie mit Schutzsuchenden ist eine Herausforderung, die gelingen kann. Sie stellt eine Befriedigung sowie eine Bereicherung auf beruflicher, menschlicher, politischer und ethischer Ebene dar. 
Interessenkonflikt R. Kronsteiner gibt an, dass kein Interessenkonflikt besteht.

Open Access. Dieser Artikel wird unter der Creative Commons Namensnennung 4.0 International Lizenz (http:// creativecommons.org/licenses/by/4.0/deed.de) veröffentlicht, welche die Nutzung, Vervielfältigung, Bearbeitung, Verbreitung und Wiedergabe in jeglichem Medium und Format erlaubt, sofern Sie den/die ursprünglichen Autor(en) und die Quelle ordnungsgemäß nennen, einen Link zur Creative Commons Lizenz beifügen und angeben, ob Änderungen vorgenommen wurden.

\section{Literatur}

Ammer, M., Kronsteiner, R., Schaffler, Y., Kurz, B., \& Kremla, M. (2013). Krieg und Folter im Asylverfahren. Eine psychotherapeutische und juristische Studie. Wien, Graz: NWV NeuerWissenschaftlicherVerlag.

Becker, D. (2006). Die Erfindung des Traumas. Verflochtene Geschichten. Freiburg: Edition Freitag.

BM.I Republik Österreich (2017). Vorläufige Asylstatistik November 2016. http://www.bmi.gv.at. Zugegriffen: 02. Jänner 2017.

Bohleber, W. (2000). Die Entwicklung der Traumatheorie in der Psychoanalyse. Psyche Sonderheft 9/10. (S. 797-839). Stuttgart: Klett-Cotta.

Dabic, M. (2010). „Darüber möchte ich nicht sprechen“. Dolmetschen für Folterüberlebende. In S. Mirzaei \& M. Schenk (Hrsg.), Abbilder der Folter. Hemayat-15 Jahre Arbeit mit traumatisierten Flüchtlingen (S. 150-160). Wien: Mandelbaum.
Felber-Villagra, N. (1995). Das Gespenst der Politik in der Psychoanalyse. In P. Möhring \& R. Apsel (Hrsg.), Interkulturelle psychoanalytische Psychotherapie(S.222-249). Frankfurt am Main: Brandes \&Apsel.

Informationsverbund Asyl und Migration e.V. (2016). Genfer Flüchtlingskonvention 1951. http:// www.asyl.net/ Gesetztestexte/Genfer Flüchtlingskonvention. Zugegriffen: 02. Jänner 12017.

Herman, J. (2006). Die Narben der Gewalt. Traumatische Erfahrungen verstehen und überwinden. Paderborn: Junfermann. Originalausgabevon 2003

Keilson, H. (2005). Sequentielle Traumatisierung bei Kindern. Untersuchung zum Schicksal jüdischer Kriegswaisen. Gießen: Psychosozial-Verlag. Originalausgabe von 1979

Kohte-Meyer, I. (2009). Funktionsstörungen des Ich und die Neuorientierung der Ich-Identität im Migrationsprozess. In Y. Erim (Hrsg.), Klinische Interkulturelle Psychotherapie. Ein Lehr- und Praxisbuch (S. 146-157). Stuttgart: Kohlhammer.

Kronsteiner, R. (2003). Kultur und Migration in der Psychotherapie. Ethnologische Aspekte psychoanalytischer und systemischer Therapie. Frankfurt am Main: Brandes \& Apsel.

Kronsteiner, R. (2010). Innere Bilder des Grauens betrachten, betrauern und verändern-Psychotherapie mit einem extrem traumatisieren Mann. In S. Mirzaei \& M. Schenk (Hrsg.), Abbilder der Folter. Hemayat-15 Jahre Arbeit mit traumatisierten Flüchtlingen (S. 72-96). Wien: Mandelbaum.

Lebowitz, L., Harvey, M. R., \& Herman, J.L. (1993). A stage-bydimension model of recovery from sexual trauma. Journal of Interpersonal Violence, 8, 378-391. 